\title{
Homeless and hungry: food insecurity in the land of plenty
}

\author{
Kevin M. Fitzpatrick ${ }^{1}$ (D) - Don E. Willis ${ }^{2}$ (1)
}

Received: 19 January 2020 / Accepted: 30 October 2020 / Published online: 4 November 2020

(C) International Society for Plant Pathology and Springer Nature B.V. 2020

\begin{abstract}
Homelessness continues to challenge service providers in the United States where $600,000+$ individuals are without their own place to stay on any given night. With significant barriers preventing access to food, homeless persons remain at risk for experiencing long-term food insecurity. As such, the primary intent of this paper was to examine specific hypotheses that explored the impact of both risks/stressors and resources on the reported food insecurity among homeless adults. Using a stratified quota sample, data were collected from homeless adults residing in two counties in Northwest Arkansas. One hundred and sixty-eight face-to-face interviews were conducted with homeless persons living in sheltered and unsheltered environments. We found a strong positive association with persons who had been arrested and reported food insecurity; anxiety symptoms and physical health symptoms were both positively related to food insecurity. Additionally, there were significant resources (access to medical services, and community connectedness) that were negatively related to food insecurity among homeless adults. Findings are discussed in the context of how important it is for service providers to have a clear picture of the health and wellbeing of their clientele, particularly as they work towards minimizing service disruption and maximizing client access to sustainable food sources.
\end{abstract}

Keywords Food insecurity $\cdot$ Homelessness $\cdot$ Risks and resources $\cdot$ Food access $\cdot$ Poverty

\section{Introduction}

Homelessness continues to challenge service providers in the United States (USA) where nearly 600,000 individuals are without their own place to stay on any given night (NAEH 2018). Millions more experience homelessness when factoring in the number of families with children that are doublingup with friends and relatives. Homelessness presents enormous challenges for both service providers and policymakers working to address basic needs and their overlap with health outcomes. These challenges are exacerbated when considering the difficulty that homeless persons have in acquiring food. With significant barriers preventing access to healthy, socially acceptable food, homeless persons remain at risk for experiencing long-term food insecurity.

Kevin M. Fitzpatrick

kfitzpa@uark.edu

1 Department of Sociology and Criminology, Old Main 211, University of Arkansas-Fayetteville, Fayetteville, AR 72701, USA

2 Department of Internal Medicine, University of Arkansas for Medical Sciences, 1125 N. College Ave., Fayetteville, AR 72703, USA
Food insecurity is defined as "access by all people at all time to enough food for an active, healthy life" (ColemanJensen et al. 2018). We build on previous work which highlighted the differentiated experience of food insecurity among the homeless (Lee et al. 2010) by assessing the role of multiple risks, stressors, and resources. We offer a unique contribution to this limited body of research on food insecurity among the homeless by highlighting the role that interaction with the criminal justice system and medical institutions playing in shaping an outcome critical to public health in the USA.

\subsection{Homelessness and food insecurity}

While the primary research on homelessness and food insecurity is limited, there are several studies that offer evidence of the expected relationship between homelessness and food insecurity, and the secondary risks (circumstances, mental and physical health) that often are the consequence of this limited access to food (Dachner and Tarasuk 2002; Goldrick-Rab et al. 2018; Smith and Richards 2008). Homeless persons have a higher risk of food insecurity than the general population (Baggett et al. 2011; Gundersen et al. 2003; Lee and Greif 
2008). Even among college students, housing insecurity, instability, and homelessness are some of the key predictors of food insecurity (Goldrick-Rab et al. 2015).

Similar to research that provides evidence that the homeless experience is vastly different across subgroups, locations, and circumstances (Fitzpatrick and LaGory 2011; Fitzpatrick and LaGory 2003; Lee et al. 2010), there is a limited body of work that has demonstrated homeless persons do not experience hunger and food insecurity uniformly. Food insecurity among the homeless is often differentiated by psychosocial coping resources such as mental health or social ties (Lee and Greif 2008; Parpouchi et al. 2016). Similar to the general population, food insecurity varies across social circumstances as well as sociodemographic subgroup membership. Building on previous research demonstrating practices which criminalize poverty, homelessness, and providing food to homeless persons (Dum et al. 2017; Gonzalez 2014; Herring 2019; Herring et al. 2020), we expected experiences of arrest to be a salient predictor in determining food insecurity among the homeless. That finding would complement Brayne's (2014) research on "surveillance and systems avoidance" which demonstrated that individuals who have been arrested are less likely to interact with medical, financial, labor market, and educational institutions, which can be critical sources for alleviating food insecurity.

Questions remain with understanding the link between health and food insecurity among homeless persons. Thus, the present study sought to better understand the mechanisms that link health indicators such as mental and physical health symptomatology to levels of food insecurity among members of a homeless population. We examined these mechanisms among a homeless population found in two counties within the Northwest region of Arkansas state, guided by a stress-process framework.

\subsection{Theoretical framework}

The stress process literature explains health disparities by highlighting unequal exposure to stressors, as well as the varying role that coping resources play to deal with that stress (Aneshensel 1992; Pearlin et al. 1981; Thoits 2006). Lee and Grief (2008) used an adaptation framework informed by the stress-process model to make sense of their findings on homelessness and hunger using data from the 1996 National Survey of Homeless Assistance Providers and Clients (NSHAPC) project. In doing so, they confirmed that exposure to the stressor of homelessness was further differentiated by both the exposure to additional stressors (e.g., alcohol, drugs, and mental health issues), as well as social and economic resources (e.g., social ties and employment). These findings complement Thoits' (2006) theoretical position that resources related to whether or not someone was able to exercise agency were both unequally distributed according to social status and highly consequential when it comes to one's ability to buffer negative stressors such as homelessness.

Our intent was to extend both the empirical and theoretical elements of this literature. We examined the role of circumstances/stressors that may inhibit one's ability to exercise the agency needed to acquire basic needs (e.g. food), as well as access personal and community resources such as mastery of fate, community connectedness, and medical services, that can enable individuals exercise of agency.

\subsubsection{Stressors}

The stress-process model helps explicate health disparities first by directing our attention to the unequal exposure of stressors across various experiences. Building on this theory, Thoits (2006) argues that attention must also be paid to the role of personal coping resources related to one's physical and mental health. These may be stressors in and of themselves, and may offer some indication of how much agency one can exercise in the face of other external stressors. Because food is a source of the energy and nutrition necessary to reproduce and replenish the body, food insecurity has a clear and direct impact on health; however, chronic physical and mental health are also strong predictors of who will experience food insecurity (Bhargava et al. 2008; Choi et al. 2017; Hanson and Olson 2012). Symptoms of poor physical health or impairment can constrain one's ability to exercise agency, particularly in built environments where there is little consideration for physical impairment or other symptoms of poor physical health. Furthermore, chronic health conditions often bring with them significant medical costs and debt that place an additional strain on household food budgets.

While food insecurity is itself a source of stress that can lead to negative mental health outcomes, mental health (e.g. anxiety) may also shape one's entry into, duration, or severity of food insecurity. As Seligman and Schillinger (2010) have demonstrated, the relationship between food insecurity and health is a reciprocal one.

The stressor model consistently alludes to the important role that life events can play in determining a host of negative health-related outcomes (Lin and Ensel 1989; Thoits 1991). Work in the general and homeless population literatures finds that these external, negative experiences can be significant correlates of poor health outcomes (Fitzpatrick and LaGory 2011; Thoits 2006). Somewhat related to the negative life experience of being arrested and having to interact with criminal justice is the circumstantial experience of living on the street unsheltered, and just how different than can be compared to persons living in some type of emergency, transition$\mathrm{al}$, or permanent supportive housing. The ongoing crisis of being without shelter is consuming and can impact a person's 
ability to acquire food. When individuals are consumed with locating a safe and secure place to sleep every night, food or healthy food may take a backseat to the immediate importance of securing shelter.

Given this literature regarding the role that stressors play in determining levels of food insecurity, we propose the following:

H1: Stressors will be associated with higher odds of food insecurity.

\subsubsection{Resources}

In addition to stressors, the stress-process model emphasizes the possibility of individuals utilizing both personal and community level resources to buffer against hardships such as homelessness. The ability to leverage personal and community resources is likely to be a key factor when it comes to differentiating the experiences/severity of food insecurity among homeless persons. Several of these resources are outlined below.

Access to medical services also plays a critical role in the cyclical relationship between food insecurity and health, as illustrated by Seligman and Schillinger (2010). Not only does access to medical care potentially prevent further health complications that often lead to or exacerbate food insecurity (Bhargava et al. 2008; Choi et al. 2017; Hanson and Olson 2012), the costs of care — and the strain on one's budget — may also increase the longer one goes without medical access and/ or treatment.

Community connectedness has been defined as a "feeling that members have of belonging, a feeling that members matter to one another and to the group, and a shared faith that members' needs will be met through their commitment to be together" (McMillan and Chavis 1986, p. 9). In other words, it is both a psychological and material resource - a link between belonging and a sense that one's needs can and will be met.

Another important psychosocial resource is mastery of fate. Mastery of fate refers to "the extent to which people see themselves as being in control of the forces that importantly affect their lives" (Pearlin et al. 1981, p. 340). It is an important "element of self" which can be a coping resource (Thoits 2006), but can also be diminished in the face of stressful life events (Pearlin et al. 1981). Past research has linked mastery of fate to quality of life among the homeless (O'Connor and Fitzpatrick 2017).

Given this literature regarding the role that personal and community resources play in determining levels of food insecurity, we propose the following:

$\mathrm{H} 2$ : Personal and community resources will be associated with lower odds of food insecurity.

\section{Methods}

The data for this study came from a 2015 survey of homeless persons $(N=168)$ in a mid-sized suburban/rural area in Northwest Arkansas. A January 2015 point-in-time (PIT) census of sheltered and unsheltered homeless persons living in both Washington and Benton Counties, Arkansas, represented the population base from which the primary sample parameters for the current study were derived (Fitzpatrick et al. 2015). The PIT has a number of limitations and was only used to identify population estimates from which the final sample was constructed. The PIT and subsequent survey interviews collected data across 31 service provider locations including unsheltered sites. Some of the service locations were food pantries and places where homeless persons typically access food on a daily basis, including day centers, community meals, and shelters.

\subsection{Participants}

Five hundred and twelve homeless adults were interviewed in the 2015 PIT, which represented the visible homeless population count on a single night in January 2015 for the two-county area. Using this population as our framework to construct the sample, we stratified the sample by county, by city, where respondents spent their last night, gender, and race. Individual sample quotas were derived based on the population estimates from the PIT and all sampling quotas were met within $\pm 2 \%$ range. Finally, respondents were randomly selected at all interview locations, either through a random number selection that used client lists, or random selection of respondents using a four-square method of selection from open spaces at interview locations like day centers, soup kitchens, food pantries, and other non-sheltered environments.

\subsection{Procedure}

Respondents were selected to participate in the survey based on meeting the above criteria for inclusion in the study. Once selected, respondents were offered a US\$20 incentive for completing the survey. Each respondent was reminded of the confidential nature of the data collection and asked to sign a consent form agreeing to participate; the primary investigator's university IRB approved the protocol. A 45-min interview was completed by 168 adults living in Washington and Benton Counties in Northwest Arkansas; fewer than 10 persons refused to participate in the survey and the response rate exceeded $92 \%$.

\subsection{Measurement}

\subsubsection{Dependent variable}

We measured food insecurity using the standard, 10-item, USDA (United States Department of Agriculture) Adult 
Food Security Module (USDA 2020). The 10-item measure was selected due to time constraints and the desire to use those questions that most appropriately captured the circumstances for homeless respondents. We began our module with the following prompt: "Thinking about your experience with food over the past year." They were then provided statements to which they could respond with "often true," "sometimes true," "never true," or "don't know or refused." They responded to the following statements: 1) I worried whether my food would run out before I got money to buy more; 2) The food that I bought just didn't last and I didn't have money to get more; 3) I couldn't afford to eat balanced meals. The remaining questions could be responded to with "yes," "no," or "don't know"- questions about how often the experience occurred were answered with "almost every month," "some months but not every month," or "only 1 or 2 months." These questions included the following: 4) In the last 12 months, did you ever cut the size of your meals because there wasn't enough money for food?; 5) How often did this happen?; 6) In the last 12 months, did you ever eat less than you felt you should because there wasn't enough money for food?; 7) In the last 12 months, were you ever hungry but didn't eat because there wasn't enough money for food?; 8) In the last 12 months, did you lose weight because there wasn't enough money for food?: 9) In the last 12 months, did you ever skip meals because there wasn't enough money for food?; 10) How often did this happen? All affirmative responses, including "some months" or "almost every month" were coded as 1 . Respondents who answered in the affirmative to 3 or more items were considered food insecure, while those who answered in the affirmative to 2 or fewer items were considered food secure (Coleman-Jensen et al. 2019).

\subsubsection{Independent variables}

Circumstantial risk Because the homeless population is generally a diverse one, and that diversity is reflected in the current sample, we elected to control for several risk variables. We were unable to find any comprehensive treatment of these variables in the food insecurity and homelessness literature. Nevertheless, we anticipated differences among certain subgroups given the extant literature on sociodemographic variation among homeless persons (Fitzpatrick 2017; Irwin et al. 2008; O'Connor and Fitzpatrick 2017). As such, we included both housing $(1=$ unsheltered $)$; $\operatorname{arrest}(1=$ Yes $)$ in the current analysis.

There are several stressors that we anticipated as being related to food insecurity among an adult homeless population. We outline, in detail, three specific factors that we believe are important and would be positively related to food insecurity.
Physical health symptoms Physical symptoms are measured using a standard collection of physical ailments typically reported by persons experiencing both acute and chronic illness. Respondents were asked if they experienced any of the 23 health symptoms in the last month prior to the interview. Health symptoms included frequent headaches, sore throat/repeated cough, dizziness, shortness of breath/difficulty breathing, coughing up blood, frequent backaches, stomach problems, broken bones, and skin problems. Respondents answered yes $=1$ or no $=0$ and the items were summed into a symptoms scale that was reliable with a Cronbach's $\alpha=.84$. Fewer than $10 \%$ of those respondents who were asked these questions said they were currently experiencing no physical health symptoms.

Anxiety symptoms Anxiety is measured using symptoms from the Brief Symptoms Inventory (BSI) subscale for anxiety (Derogatis and Spencer 1982). There were considerable time limits placed on us during the interview and thus we only measured the presence of symptoms (not intensity). The symptom index was comprised of the following symptoms associated with high levels of manifest anxiety, including panic attacks, terror, tension, and apprehension. Each question had dichotomous responses and the scale was the summation of these responses. Scores range from 0 to 2 and typically scores less than 2 are considered mild; scores of 2 and above are moderate and noteworthy. Of the 6 different anxiety symptoms that were assessed, $23 \%$ reported four or more symptoms in the past month. The scale was reliable with a Cronbach's $\alpha=.84$.

Weight status Weight status was determined using the Centers for Disease Control (CDC) charts that provide estimates based on gender and age (Centers for Disease Control 2017). Respondents were classified based on these national standards: 1) Underweight (BMI $<5$ th percentile); 2) Normal (BMI 5th to 84th percentile); 3) Overweight (BMI 85th to 94th percentile); and 4) Obese (BMI $>95$ th percentile). Respondents who were underweight were removed from this particular analysis and we combined overweight and obese $(64 \%)$ into a single category (value $=1$ ) and normal BMI $(33 \%)$ in a second category (value $=0$ ). The number of persons in our sample who were actually underweight was small $(<5)$. Their impact on this analysis was examined and determined to not influence any of the results. Thus it was much easier to simply remove them from the analysis and maintain the integrity of the dichotomous dependent variable.

There are a number of resources (social and psychological) that we hypothesized as being related to food insecurity among an adult homeless population. We outline, in detail three specific resources that we expected to be negatively related to food insecurity. 
Community connectedness We measured connectedness using the Inclusion of Community in Self (ICS) scale, which is a single-item picture measure that consists of six pairs of overlapping circles. This measure, as an extension and variation of the Inclusion of Others in Self Scale (IOS) (Aron et al. 1992), and a Psychological Sense of Community (PSOC) (McMillan and Chavis 1986), seemed particularly apropos in the present context for studying homeless persons and their degree of connectedness to community.

Figure 1 provides the graphic that respondents were asked to characterize where they saw themselves in terms of personal connection to their community. In Fig. 1, the circles are of equal size and begin with two circles that are not touching one another and move left to right in varying degrees of closeness between the circles. The final set of circles are fully integrated with one circle essentially being inside of the other circle. One circle represents the "self" and the other circle "community." Participants were asked to look at the Venn diagrams and respond with a number associated with a particular circle set that best described their relationship to the community at large.

With little or no additional explanation provided by interviewers, the majority of respondents had little difficulty responding to the purposely vague construct of "community at large." If additional explanation was needed, interviewers offered guidance by telling them that they were to think about the community as "all those persons, places, and things that made up the larger community". No specific group or subgroup was used as a referent and interviewers reported very few problems with obtaining what they believed to be reliable responses.

Mastery of fate Another resource variable (psychological), was assessed using a 7-item Likert scale that asked respondents about their ability to control their environment. We used a scale developed by Pearlin and Schooler (1978) where higher scores indicate greater mastery and locus of control. Scores are from 0 to 21 with responses ranging from 0 (strongly disagree) to 3 (strongly agree). For the current sample, the scale is reliable with a Cronbach's $\alpha=.76$.

Access to medical services Respondents were asked a number of questions related directly to their access or lack thereof to medical services. For this particular indicator we selected three specific dichotomies and each of the variables had responses that were coded as Yes $=1$ and $\mathrm{No}=0$. Respondents were asked: 1) if in the last year were they able to see a medical professional?; 2) specifically, did they see a dentist in the last year?; and 3) were they able to receive treatment for some medical condition?.

\subsection{Analytic strategy}

Descriptives are presented to help the reader orient to the sample characteristics that we report. The primary focus of the analysis was to examine the relationships of circumstantial risk, stressors, and resources with food insecurity using hierarchical logistic regression. We entered each of these sets of variables into the regression models as blocks to demonstrate both main effects and their collective influence on food insecurity. While a typical study of food insecurity might also include a set of sociodemographic controls, our early analyses found that factors like race, ethnicity, and sex, were not differentiating the odds of being food insecure among the homeless; thus, our analytic strategy focused on circumstantial risks, stressors, and resources. The logistic analysis presented in Table 2, shows individual odds ratios (OR) when larger than one, suggests an increased odds in food insecurity and when odds ratios are less than one, a decreased odds in food insecurity. Additionally, 95\% confidence intervals $(\mathrm{CI})$ are presented to assess the statistical significance of individual variables, and also the "strength" of that significance given the range of confidence interval reported. Finally, a descriptive summary measure that assesses the strength of each model (Nagelkerke $\mathrm{R}^{2)}$ is also presented. Three successive models were assessed with each one reflecting the addition of a set of variables that we were able to assess. The cumulative explained variation that is reached in Model 3 reflects the strength of the model when all the variables were included in the equation.

\section{Results}

In Table 1 we present descriptive data on the variables used in the analysis of 168 homeless adults in Northwest Arkansas. The sample of homeless adults was predominantly male, over 40 , and white, with a high school education. On average, homeless adults answered in the affirmative to nearly six (5.92) out of the ten food insecurity questions. Not a single person in this sample scored a zero on the food insecurity scale - the score necessary to be considered completely food secure-and $70 \%$ scored three or higher-the threshold
Fig. 1 Inclusion of Community in the Self Scale

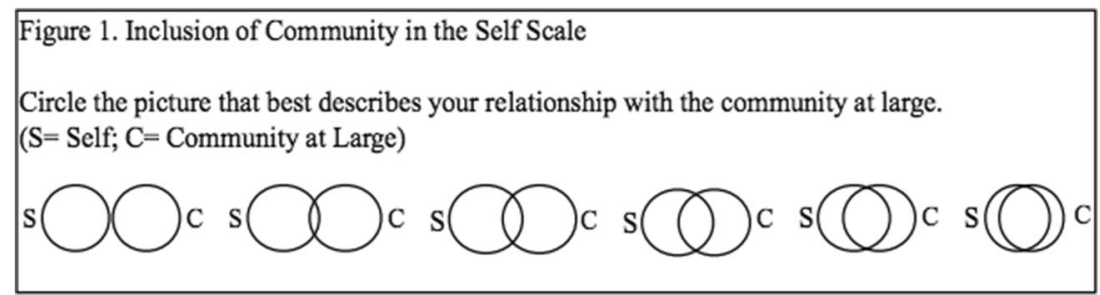


Table 1 Descriptive statistics for model variables $(n=158)$

\begin{tabular}{llll}
\hline & $\%$ & Mean & S.D. \\
\hline Dependent Variable & & & \\
$\quad$ Food Secure (<3) & $30 \%$ & & \\
Food Insecure (3+) & $70 \%$ & & \\
Circumstantial Risk & & & \\
Arrested (1 = Yes) & $72 \%$ & & \\
Housing (1 = Unsheltered) & $10 \%$ & & \\
Stressors & & & .88 \\
Physical Health Symptoms & & 6.0 & \\
Anxiety Symptoms & & 2.4 & 2.1 \\
Weight Status (1 = Overweight/Obese) & $67 \%$ & & \\
Resources & & & \\
Community Connectedness & & 2.9 & .89 \\
Mastery of Fate & & 12.8 & 3.6 \\
Medical Access & & 1.4 & .90 \\
\hline
\end{tabular}

typically used to identify someone as categorically food insecure. This is a much higher prevalence of food insecurity than the $11 \%$ who are considered to be food insecure in the overall US population (Coleman-Jensen et al. 2019). The average anxiety symptom score was high relative to the general population. Homeless respondents on average reported at least six physical health symptoms, and had relatively low mastery of fate scores. Two-thirds of the sample of respondents was either overweight or obese, which is very close to the $71 \%$ who are estimated to be overweight or obese in the general US population (CDC 2020). Community connection scores were slightly lower than average.

Table 2 provides the results for the models with the food insecurity dichotomy regressed on circumstantial risks, stressors, and resources in four separate models with each group added in a hierarchical fashion. The consistent circumstance variable of being arrested was significant $(p<0.05)$ in all three models. Homeless respondents that had experienced arrest were 2.5 to nearly 3 times more likely to report food insecurity compared to those homeless respondents that had not experienced arrest. While the experience of being unsheltered was not significant, we interpret that result with some caution. The number of unsheltered respondents in the sample was low, which may be related to the lack of statistical significance.

Additionally, all of the stressor variables were positively related to food insecurity as originally hypothesized. Higher levels of anxiety symptomatology and physical health symptoms were the two stressor variables that were statistically significant $(\mathrm{p}<0.05)$. Anxiety symptoms increased the odds of food insecurity by nearly $30 \%$, while physical health symptoms increased the odds by $13 \%$. The model that included the circumstantial risk and the stressors variables was significant with Nagelkerke $=0.19$. These stressors compound already desperate circumstances for many homeless adults and clearly impact their access to food, and/or their consistent access to healthy foods.

In addition to these negative stressors, we added a set of important resources that we expected to be negatively related to food insecurity among this homeless population. Medical access and community connectedness were statistically significant and in the expected (negative) direction. Community connectedness lowered the odds of food insecurity among the homeless by almost $25 \%$, and medical access lowered the odds by approximately $72 \%$. The psychological resource, mastery, was not significant and the final model as a whole was significant with a Nagelkerke $=0.32$.

\section{Discussion}

Extant research has demonstrated that homelessness is not experienced uniformly across all population subgroups and circumstances (O'Connor and Fitzpatrick 2017), and that food insecurity among the homeless is differentiated further by the resources available to them and the additional risks they face (Lee and Greif 2008; Parpouchi et al. 2016). The present study sought to examine the differentiated experience of homelessness and further explore some of the mechanisms which might help explain why some homeless persons' experience food insecurity while others do not, and why some homeless persons experience food insecurity with more severity than others. Our findings highlight how the interactions of homeless persons with two key institutions - the criminal justice system and medical institutions - play a significant role in shaping their likelihood to experience food insecurity in Arkansas. Specifically, arrests nearly tripled the odds of food insecurity for the homeless in this study, while medical access reduced the likelihood of food insecurity by more than $70 \%$. These findings also raise additional questions for new lines of research regarding exactly how those institutions heighten or reduce the chances of food insecurity among the homeless. We suggest that future studies explore further the possibility of what Brayne (2014) has termed "surveillance and systems avoidance," in which individuals who have been arrested tend to avoid a whole host of institutions (e.g., financial, medical) which might be critical to reducing their odds of food insecurity.

Our findings underscore the importance of the health-food relationship impacting America's most vulnerable. This population is often receiving temporary food relief from pantries that typically are not providing access to healthy foods, but snacks, desert items, and other items that are highly processed, high in sugar content, and provide little nutritional substance. Again, food insecurity and the risk for negative health outcomes related to this insecurity is typically not as much about 
Table 2 Logistic regression models for food insecurity among homeless adults $(n=158)$

\begin{tabular}{|c|c|c|c|c|c|c|}
\hline & \multicolumn{2}{|c|}{ Model 1} & \multicolumn{2}{|c|}{ Model 2} & \multicolumn{2}{|l|}{ Model 3} \\
\hline & OR & $95 \% \mathrm{CI}$ & OR & $95 \% \mathrm{CI}$ & OR & $95 \% \mathrm{CI}$ \\
\hline \multicolumn{7}{|l|}{ Circumstantial Risk } \\
\hline Arrested (1 = Yes) & $2.44 *$ & $1.16-5.14$ & $2.93 * *$ & $1.28-6.65$ & $2.71 *$ & $1.14-6.49$ \\
\hline Housing ( 1 = Unsheltered $)$ & 2.62 & $.562-12.25$ & 3.35 & $.672-16.67$ & 2.91 & $.551-15.39$ \\
\hline \multicolumn{7}{|l|}{ Stressors } \\
\hline Physical Health Symptoms & & & 1.09 & $.992-1.21$ & $1.13 *$ & $1.01-1.25$ \\
\hline Anxiety Symptoms & & & $1.30 *$ & $1.06-1.59$ & $1.29 *$ & $1.03-1.62$ \\
\hline Weight Status ( $1=$ Overweight/Obese $)$ & & & .667 & $.295-1.51$ & .743 & $.310-1.78$ \\
\hline \multicolumn{7}{|l|}{ Resources } \\
\hline Community Connectedness & & & & & $.747 *$ & $.572-.975$ \\
\hline Mastery of Fate & & & & & .987 & $.876-1.11$ \\
\hline Medical Access & & & & & $.284 * *$ & $.284-.746$ \\
\hline Constant & 3.20 & & 1.31 & & 10.16 & \\
\hline Nagelkerke $\mathrm{R}^{2}$ & $.071 *$ & & $.191 * *$ & & $.320 * * *$ & \\
\hline
\end{tabular}

$p<.05^{*} ; p<.01^{* *} ; \mathrm{OR}=$ odds ratios; $\mathrm{CI}=$ confidence intervals

a lack of food, as it is about the limited access to healthy foods and socially acceptable ways of accessing food.

The findings reported here are consistent with earlier work that reports high risk for food insecurity among the homeless population generally, and the chronic, unsheltered homeless population more specifically (Baggett et al. 2011; Kushel et al. 2005). In addition, the link between health risks and food insecurity that is reported elsewhere is, in part, confirmed here; yet, this subject still requires a more comprehensive examination of the intersection of food access, nutrition, and negative health outcomes among high-risk homeless adults.

\subsection{Study limitations}

Despite the present study's findings that highlight the importance of health-related stressors, social and psychological resources for both the food insecurity literature generally, and the homeless literature specifically, we note some important limitations. First, the research was limited by the exclusive use of self-report measures, which could be confounded by a common method variance, and self-report bias that could be improved with multiple methods of reporting, thus improving the validity of findings. Furthermore, some measures such as medical access may represent more than barriers to medical care, but could also indicate personal prioritization given that the questions asked only whether they saw certain medical professionals or received care for something. Additionally, this was a 45-min and sometimes even longer interview. We acknowledge the potential for interviewee/interviewer fatigue, which may have implications for some of the responses that were coded and used in the current analysis.
Second, the data used here are cross-sectional and while we can establish the nature of the relationships between sets of independent and dependent variables, these findings do not lend themselves to causal conclusions. Longitudinal data would vastly improve our ability to make these causal connections, but nevertheless this particular subgroup of underserved are extremely transient and would represent a major challenge to follow over an extended period of time. We also recognize the bi-directionality of some of these relationships as well as the counter/alternative arguments that are made for why a particular variable may or may not be important to our analysis.

Third, the use of single measures for many of the variables used in the analysis is a further limitation. Future research could include both a wider range of variables and a series of more complex models to further explicate the interrelationships between health stressors, resources and food insecurity among homeless persons.

Finally, we recognize that these findings are placed in the context of a single sample of homeless adults living in a particular region of the South-Central part of the United States. Nevertheless, this sample was not a convenience sample: the sampling design followed strict protocols that guaranteed randomness and representativeness. As highlighted earlier in the paper, all the quotas important to sampling design were met, ensuring generalizability to the larger homeless population of this region.

While these are significant limitations, they are balanced out by several strengths of this research design and the substantive contribution of the findings. This study adds to a limited body of research regarding food insecurity among 
the homeless, and provides new insight into why some homeless struggle even more severely than others in terms of access to basic needs.

\subsection{Covid-19 implications}

While our data collection took place five years ago, much of our work, these findings, and its relevance to food insecurity being unevenly distributed across socially vulnerable subgroups remain relevant. Additionally, the recent novel coronavirus (Covid-19) pandemic has alerted us to the fragility of our food distribution system both in the United States and world-wide, with stark reminders that those persons who were experiencing food insecurity prior to the pandemic, are potentially at greater risk not only for greater food insecurity but negative physical and mental health consequences too (Béné 2020; Fitzpatrick et al. 2020a and b; Tsai and Wilson 2020). This pandemic has amplified the problem of food access, food safety, food distribution, and the importance in identifying weaknesses in our local food systems. Clearly, new and innovative strategies will be required to address these weaknesses as we face future shocks to our food systems as a result of both natural disasters and public health crises (Barker and Russell 2020; Devereux et al. 2020; Fitzpatrick et al. 2020a, b).

\section{Conclusions}

This study utilized a stress-process framework to investigate factors that distinguish the experience of food insecurity among homeless adults. Our findings support three primary conclusions.

First, health stressors are associated with higher levels of food insecurity. Both physical and mental health symptoms were significantly associated with higher levels of food insecurity. These findings are consistent with the model developed by Seligman and Schillinger (2010) which posits a reciprocal relationship with health and food insecurity, wherein they both influence each other, as well as several empirical studies which demonstrate similar findings (Choi et al. 2017). The implication here is that, just as addressing food insecurity impacts health care systems by alleviating the long list of negative health outcomes it is associated with, health care policy must play a significant role in addressing issues of food insecurity.

Second, resources are associated with lower levels of food insecurity. Community connectedness and access to medical services were associated with lower levels of food insecurity. This is consistent with previous work showing both social and economic resources to be significant predictors of food insecurity (Bhargava et al. 2008; Fitzpatrick et al. 2020b; Heflin et al. 2005; Willis 2019; Willis and Fitzpatrick 2019). Thus, we find support for both the $\mathrm{H} 1$ and $\mathrm{H} 2$ series of hypotheses.
Lastly, these findings highlight the role of two major social and institutional forces in shaping the experience of homelessness. The two variables that influenced the odds of food insecurity the most were arrests and medical access - arrests increasing odds nearly threefold, and medical access lowering them by almost $75 \%$. Based on these results, we conclude that the criminal justice and health care systems in the USA play important roles in shaping everyday access to healthy food among homeless persons. Moreover, these two systems highlight drastically diverging methods of addressing homelessness - one being a punitive or criminalizing approach, the other an approach with care - and this divergence is indicated in part by their opposite impacts on food insecurity among this population. While this study is not the first to link the experiences of homelessness to these two diverging systems, the degree to which an arrest or access to medical care can shape the odds of homeless persons meeting their basic human need for food is a unique and striking contribution. These findings demonstrate what the criminalization of homelessness can mean for food insecurity, as well as the potential for reducing the risk of food insecurity among homeless persons through targeted policies that can expand their access to medical care.

Acknowledgements The Wal-Mart Foundation funded this research. KF collected the data, conceptualized the study and current manuscript, outlined the analysis, and wrote extensive sections of the paper and its revisions. DW helped with the conceptualization and food insecurity focus, helped with the analysis and the writing up of results and construction of tables, as well as wrote significant sections of the paper and its revisions. We would like to thank the anonymous reviewers and editorial team for their suggested improvements of the manuscript. Finally, our heartfelt thanks to all of the interviewees that took the time to respond to us despite their precarious and uncertain circumstances that they were facing on a daily basis.

Availability of data Upon request.

\section{Compliance with ethical standards}

Conflict of interest The authors declared that they have no conflict of interest.

Code availability Upon request.

\section{References}

Aneshensel, C. S. (1992). Social stress: Theory and research. Annual Review of Sociology, 18(1), 15-38.

Aron, A., Aron, E. N., \& Smollan, D. (1992). Inclusion of other in the self scale and the structure of interpersonal closeness. Journal of Personality and Social Psychology, 63(4), 596-612.

Baggett, T. P., Singer, D. E., Rao, S. R., O’Connell, J. J., Bharel, M., \& Rigotti, N. A. (2011). Food insufficiency and health services utilization in a national sample of homeless adults. Journal of General Internal Medicine, 26(6), 627-634. 
Barker, M., \& Russell, J. (2020). Feeding the food insecure in Britain: Learning from the 2020 COVID-19 crisis. Food Security, 12(4), $865-870$.

Béné, C. (2020). Resilience of local food systems and links to food security - A review of some important concepts in the context of COVID-19 and other shocks. Food Security, 12(4), 805-822.

Bhargava, A., Jolliffe, D., \& Howard, L. L. (2008). Socio-economic, behavioural and environmental factors predicted body weights and household food insecurity scores in the early childhood longitudinal study-kindergarten. British Journal of Nutrition, 100(2), 438-444.

Brayne, S. (2014). Surveillance and system avoidance: Criminal justice contact and institutional attachment. American Sociological Review, 79(3), 367-391.

Centers for Disease Control and Prevention (2017). About Adult BMI | Healthy Weight | CDC. https://www.cdc.gov/healthyweight/ assessing/bmi/adult bmi/index.html. Accessed 31 December 2018.

Centers for Disease Control and Prevention (2020). FastStats. https:// www.cdc.gov/nchs/fastats/obesity-overweight.htm. Accessed 17 September 2020.

Choi, S. K., Fram, M. S., \& Frongillo, E. A. (2017). Very low food security in US households is predicted by complex patterns of health, economics, and service participation. The Journal of Nutrition, 147(10), 1992-2000.

Coleman-Jensen, A., Rabbitt, M. P., Gregory, C. A., \& Singh, A. (2018). Household food security in the United States in 2017 (no. ERR256). U.S. Department of Agriculture, Economic Research Service. http://www.ers.usda.gov/publications/pub-details/?pubid= 90022. Accessed 18 January 2019.

Coleman-Jensen, A., Rabbitt, M. P., Gregory, C. A., \& Singh, A. (2019). Household food security in the United States in 2018 (no. 270). Economic Research Service. http://www.ers.usda.gov/publications/ pub-details/?pubid=94848. Accessed 23 November 2019.

Dachner, N., \& Tarasuk, V. (2002). Homeless "squeegee kids": Food insecurity and daily survival. Social Science and Medicine, 54(7), 1039-1049

Derogatis, L. R., \& Spencer, P. (1982). Administration, scoring and procedures: BSI- manual-I. Department of Psychiatry and Behavioral Sciences, Baltimore, MD.

Devereux, S., Béné, C., \& Hoddinott, J. (2020). Conceptualising COVID-19's impacts on household food security. Food Security, 12(4), 769-772.

Dum, C. P., Norris, R. J., \& Weng, K. (2017). Punishing benevolence: The criminalization of homeless feeding as an act of state harm. Critical Criminology, 25(4), 483-506.

Fitzpatrick, K. M. (2017). Understanding community connectedness among homeless adults. Journal of Community Psychology, 45(3), 426-435.

Fitzpatrick, K. M., \& LaGory, M. (2003). "Placing" health in an urban sociology: Cities as mosaics of risk and protection. City and Community, 2(1), 33-46.

Fitzpatrick, K. M., \& LaGory, M. (2011). Unhealthy cities: Poverty, race, and place in America. New York, NY: Routledge.

Fitzpatrick, K., Collier, S., \& O'Connor, G. (2015). A comprehensive examination of homelessness in Washington and Benton counties. Fayetteville, AR: Community and Family Institute, University of Arkansas.

Fitzpatrick, K., Harris, C., \& Drawve, G. (2020a). Assessing U.S. food insecurity in the United States during COVID-19 pandemic. Fayetteville, AR: Community and family institute. Department of Sociology and Criminology, University of Arkansas, Fayetteville, AR. https://fulbright.uark.edu/departments/sociology/researchcenters/community-family-institute/ resources/community-andfamily-institute/assessing-food-insecurity-brief.pdf. Accessed 19 August 2020.

Fitzpatrick, K., Harris, C., Drawve, G., \& Willis, D. (2020b). Food insecurity in a time of fear and panic: Assessing risks and protections among a national sample of adults during the COVID-19 pandemic. Journal of Hunger and Environmental Nutrition. Forthcoming.
Goldrick-Rab, S., Broton, K., \& Eisenberg, D. (2015). Hungry to learn: Addressing food \& housing insecurity among undergraduates. Wisconsin Hope Lab, 1-25.

Goldrick-Rab, S., Richardson, J., Schneider, J., Hernandez, A., \& Cady, C. (2018). Still hungry and homeless in college (p. 52). Wisconsin Hope lab.

Gonzalez, M.-T. (2014). Hunger, poverty, and the criminalization of food sharing in the new gilded age. American University Journal of Gender, Social Policy and the Law, 23, 231.

Gundersen, C., Weinreb, L., Wehler, C., \& Hosmer, D. (2003). Homelessness and food insecurity. Journal of Housing Economics, 12(3), 250-272.

Hanson, K. L., \& Olson, C. M. (2012). Chronic health conditions and depressive symptoms strongly predict persistent food insecurity among rural low-income families. Journal of Health Care for the Poor and Underserved, 23(3), 1174-1188.

Heflin, C. M., Siefert, K., \& Williams, D. R. (2005). Food insufficiency and women's mental health: Findings from a 3-year panel of welfare recipients. Social Science and Medicine, 61(9), 1971-1982.

Herring, C. (2019). Complaint-oriented policing: Regulating homelessness in public space. American Sociological Review, 84(5), 769-800.

Herring, C., Yarbrough, D., \& Marie Alatorre, L. (2020). Pervasive Penality: How the criminalization of poverty perpetuates homelessness. Social Problems, 67(1), 131-149.

Irwin, J., LaGory, M., Ritchey, F., \& Fitzpatrick, K. (2008). Social assets and mental distress among the homeless: Exploring the roles of social support and other forms of social capital on depression. Social Science and Medicine, 67(12), 1935-1943.

Kushel, M. B., Hahn, J. A., Evans, J. L., Bangsberg, D. R., \& Moss, A. R. (2005). Revolving doors: Imprisonment among the homeless and marginally housed population. American Journal of Public Health, 95(10), 1747-1752.

Lee, B. A., \& Greif, M. J. (2008). Homelessness and hunger. Journal of Health and Social Behavior, 49(1), 3-19.

Lee, B. A., Tyler, K. A., \& Wright, J. D. (2010). The new homelessness revisited. Annual Review of Sociology, 36(1), 501-521.

Lin, N., \& Ensel, W. M. (1989). Life stress and health: Stressors and resources. American Sociological Review, 54(3), 382-399.

McMillan, D. W., \& Chavis, D. M. (1986). Sense of community: A definition and theory. Journal of Community Psychology, 14(1), 6-23.

NAEH. (2018). State of homelessness. National Association to End Homelessness. https://endhomelessness.org/homelessness-inamerica/homelessness-statistics/state-of-homelessness-report/. Accessed 23 November 2019.

O'Connor, G., \& Fitzpatrick, K. (2017). Do place and time make a difference? Examining quality of life among homeless persons in Northwest Arkansas and Birmingham. Community Mental Health Journal, 53(3), 334-339.

Parpouchi, M., Moniruzzaman, A., Russolillo, A., \& Somers, J. M. (2016). Food insecurity among homeless adults with mental illness. PLoS One, 11(7), e0159334.

Pearlin, L. I., \& Schooler, C. (1978). The structure of coping. Journal of Health and Social Behavior, 19(1), 2-21.

Pearlin, L. I., Menaghan, E. G., Lieberman, M. A., \& Mullan, J. T. (1981). The stress process. Journal of Health and Social Behavior, 22(4), 337-356.

Seligman, H. K., \& Schillinger, D. (2010). Hunger and socioeconomic disparities in chronic disease. New England Journal of Medicine, 363(1), 6-9.

Smith, C., \& Richards, R. (2008). Dietary intake, overweight status, and perceptions of food insecurity among homeless Minnesotan youth. American Journal of Human Biology, 20(5), 550-563.

Thoits, P. A. (1991). On merging identity theory and stress research. Social Psychology Quarterly, 54(2), 101-112.

Thoits, P. A. (2006). Personal agency in the stress process. Journal of Health and Social Behavior, 47(4), 309-323. 
Tsai, J., \& Wilson, M. (2020). COVID-19: A potential public health problem for homeless populations. The Lancet Public Health, 5(4), e186-e187.

USDA. (2020). USDA ERS - Survey Tools. https://www.ers.usda.gov/ topics/food-nutrition-assistance/food-security-in-the-us/surveytools/\#adult. Accessed 12 October 2020.

Willis, D. E. (2019). Feeding the student body: Unequal food insecurity among college students. American Journal of Health Education, $50(3), 167-175$.

Willis, D. E., \& Fitzpatrick, K. M. (2019). Food insecurity and social capital among middle school students. Youth and Society, 51(8), $1127-1144$

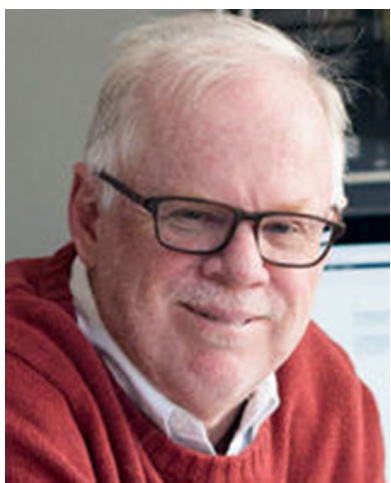

Kevin M. Fitzpatrick, $\mathrm{PhD}$, is a community sociologist with 35 years of experience as a researcher, consultant, and advocate. Kevin is a University Professor in the Department of Sociology and Criminology at the University of Arkansas. His work primarily focuses on health and the intersection of place as it impacts disadvantaged populations, homeless persons, persons facing the aftermath of a natural disaster, and low-income minority adolescents. Food insecurity has been an ongoing part of his work that is looking at the outcomes of disadvantage, limited access, and a continued lack of affordability to healthy foods in low-income settings.

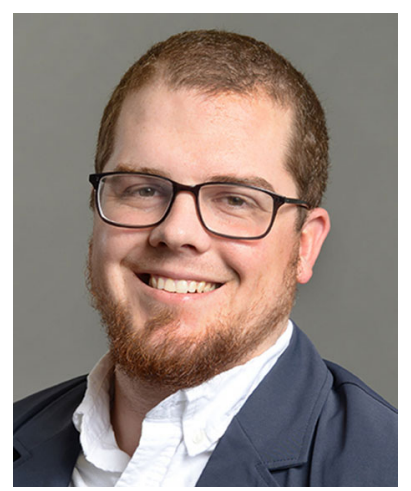

Don E. Willis is an Assistant Professor of Sociology and Poverty Studies Coordinator at the University of Arkansas at Little Rock. His research examines the intersections of poverty, inequality, health, and food. Consistent throughout his work is a critical eye towards the social patterning of life chances. 